\title{
Elucidation of the Mechanism for Maintaining Ultrafast Domain Wall Mobility Over a Wide Temperature Range
}

Sina Ranjbar ( $\sim$ sina.ranjbar@toyota-ti.ac.jp )

Toyota Technological Institute https://orcid.org/0000-0001-9435-3584

\section{Satoshi Sumi}

Toyota Technological Institute

\section{Sota Kambe}

Toyota Technological Institute

\section{Pham Van Thach}

Institute of Materials Science, Vietnam Academy of Science and Technology

\section{Kenji Tanabe}

Toyota Technological Institute

\section{Hiroyuki Awano}

Toyota Technological Institute

\section{Article}

Keywords: 5G Communication Standard, Racetrack Memory, Joule Heat Generation Distribution, Short Pulse Current

Posted Date: May 12th, 2021

DOI: https://doi.org/10.21203/rs.3.rs-401385/v1

License: (9) This work is licensed under a Creative Commons Attribution 4.0 International License. Read Full License

Version of Record: A version of this preprint was published at Materials Advances on July 29th, 2022. See the published version at https://doi.org/10.1039/D2MA00273F. 


\title{
Elucidation of the Mechanism for Maintaining Ultrafast Domain Wall Mobility Over a Wide Temperature Range
}

\author{
S. Ranjbar ${ }^{1}$, S. Sumi ${ }^{1}$, S. Kambe ${ }^{1}$, P. V. Thach ${ }^{1,2}$, K. Tanabe ${ }^{1}$, H. Awano ${ }^{1}$ \\ ${ }^{1}$ Toyota Technological Institute, Nagoya 468-8511, Japan \\ ${ }^{2}$ Institute of Materials Science, Vietnam Academy of Science and Technology, 18 \\ Hoang Quoc Viet, Hanoi, Vietnam
}

To realize a data rate of 20 Gbps in the communication standard 5G with a racetrack memory, it is crucial to stably recognize a domain-wall (DW) velocity ( $\left.v_{\mathrm{DW}}\right)$ of $1200 \mathrm{~m} / \mathrm{s}$ when the minimum bit length is $60 \mathrm{~nm}$. However, general $v_{\mathrm{Dw}}$ is as slow as about 100 m/s. Recent reports indeed showed that the fast DW motion occurs using an in-plane external magnetic field however, this mechanism is unsuitable because the rear-edge $v_{\text {DW }}$ decelerated, which contrary to the front-edge of DW velocity. Therefore, we designed magnetic wires by bringing the g values of rare-earth and transition-metals close to each other and suppressing the Joule heat generation distribution due to short pulse current, we successfully demonstrated the $v_{\mathrm{DW}}$ of $1200 \mathrm{~m} / \mathrm{s}$ in a wide temperature range without using an external magnetic field. Moreover, the current density $(J)$ is low, and the DW mobility $\left(v_{\mathrm{DW}} / \boldsymbol{J}\right)$ is significantly improved 10-times over a wide temperature range compared to other reports. 
Spintronics applications are being extensively followed for high-performance logic computing technologies and racetrack memories ${ }^{3,4,5,1}$. The principal difficulties are to reach small bits ${ }^{6}$, high thermal stability, and track them at high speed ${ }^{7}$. In ferromagnetic materials, low coercivity $\left(H_{\mathrm{c}}\right)$ is an advantage, however, large critical current density $\left(J_{\mathrm{c}}\right)$, bit size, stray field interactions limit, and precessional dynamic limit of the operating speeds are the substantial limitations in ferromagnetic materials ${ }^{8,9,10}$. Moreover, antiferromagnets show a lack of stray fields with a high packing density ${ }^{11,8,12}$. Nevertheless, manipulating and recognizing magnetic state and spin textures of antiferromagnetic are challenging ${ }^{13,14,15}$. To overcome this deficiency an alternative strategy is using amorphous rare earth transition metals (RE-TM) ferrimagnetic systems. Ferrimagnetic materials consist of rare-earth (RE) and transition metal (TM) compounds in which the RE and the TM sublattices are aligned antiparallel with each other and reduce the net angular and magnetic moments. Therefore, ferrimagnets such as GdFeCo alloy can be easily detected using optical and electrical instruments owing to the $3 \mathrm{~d}$ electrons of the transition metal sublattices ${ }^{16,17}$. Additionally, the perpendicular ferrimagnetic wires show smaller $\left(J_{\mathrm{c}}\right)$ than those in-plane wires ${ }^{18,19}$, due to low saturation magnetization $M_{\mathrm{s}}{ }^{20}$.

Theories and experimental results have predicted that the fast current-induced domain wall motion (CIDWM) appears near the angular momentum compensation temperature for ferrimagnetic wires ${ }^{3,21,22}$. To achieve the $20 \mathrm{Gbps}$ for the $5 \mathrm{G}$ application with racetrack memory, domain wall (DW) motion should be realized, with a speed of $1200 \mathrm{~m} / \mathrm{s}$ and a minimum bit length of $60 \mathrm{~nm}^{6}$ in magnetic wires. Hence, the most significant issue is the fast and stable DW velocity over $1200 \mathrm{~m} / \mathrm{s}$. The idea behind our experiments, illustrated in Fig. 
$1(\mathrm{a}-\mathrm{c})$, is based on predictions of the DW motion for various RE-TM combinations, which is corresponding to the g values of $\mathrm{RE}^{23}$ and $\mathrm{TM}^{24}$ metals. Figure 1(a) shows that the angular momentum compensation temperature $\left(T_{\mathrm{AMC}}\right)$ of the $\mathrm{TbCo}$ is far from the magnetic momentum compensation temperature $\left(T_{\mathrm{MC}}\right)$, therefore $\mathrm{DW}$ velocity is slow at any temperature. In the case of GdCo, both $T_{\mathrm{AMC}}$ and $T_{\mathrm{MC}}$ become near, the DW velocity increase, however, it is still below $1200 \mathrm{~m} / \mathrm{s}$ for the 20 Gbps request. On the other hand, the $T_{\mathrm{AMC}}$ of the GdFeCo is very near to the $T_{\mathrm{MC}}$, accordingly, DW velocity would be accelerated over $1200 \mathrm{~m} / \mathrm{s}$ in a wide temperature range.

Furthermore, we compared our work with previous reports in Table 1. These results have motivated us to analyze the mechanism of DW motion between $T_{\mathrm{AMC}}$ and $T_{\mathrm{MC}}$ for compensated ferrimagnet. To verify the prediction of DW motion between $T_{\mathrm{AMC}}$ and $T_{\mathrm{MC}}$, we performed systematic experiments for CIDWM in $\mathrm{Gd}_{\mathrm{x}}\left(\mathrm{Fe}_{88} \mathrm{Co}_{12}\right)_{100-\mathrm{x}}$ ferrimagnetic wires. We exhibit that, by employing the Gd-FeCo ferrimagnet, the DW velocity reaches its maximum $(2000 \mathrm{~m} / \mathrm{s})$ between magnetic $\left(x_{\mathrm{MC}}\right)$ and angular momentum $\left(x_{\mathrm{AMC}}\right)$ compensation composition. Then we analyzed the temperature distribution of the magnetic wire by applying the current $(J)$, and found that the DW velocity increases when the temperature difference between the center and the edge of the magnetic wire is small. We also found that the DW velocity is the fastest for magnetic wires where the $T_{\mathrm{AMC}}$ and $T_{\mathrm{MC}}$ are close to each other and $T_{\text {AMC }}$ is slightly higher than room temperature. As a result, a DW velocity of $1200 \mathrm{~m} / \mathrm{s} \mathrm{can}$ be achieved in a wide temperature range, and DW mobility $\left(v_{\mathrm{DW}} / J\right)$ is improved more than 10-times compared to other reports. 


\section{Design and sample characterization}

We initially discern the magnetic properties of $\mathrm{Gd}_{\mathrm{x}}\left(\mathrm{Fe}_{88} \mathrm{Co}_{12}\right)_{100-\mathrm{x}}$ films. Figure 2(a) exhibits the hysteresis loops were measured using the Polar-Magneto-Optical Kerr Effect (PMOKE) at room temperature. The polarity of the Kerr rotation $\left(\theta_{\mathrm{K}}\right)$ signals changes between $\mathrm{Gd}_{24}\left(\mathrm{Fe}_{88} \mathrm{Co}_{12}\right)_{26}$ and $\mathrm{Gd}_{25}\left(\mathrm{Fe}_{88} \mathrm{Co}_{12}\right)_{75}$ samples are consistent with a transition from being FeCo dominated to being Gd dominated in the magnetic moment. Figure 2(b) shows the $M_{\mathrm{s}}$ and $H_{\mathrm{c}}$ of deposited $\mathrm{Gd}_{\mathrm{x}}\left(\mathrm{Fe}_{88} \mathrm{Co}_{12}\right)_{100-\mathrm{x}}$ films at different compositions. The saturation magnetization $\left(M_{\mathrm{s}}\right)$ of $\mathrm{Gd}_{\mathrm{x}}\left(\mathrm{Fe}_{88} \mathrm{Co}_{12}\right)_{100-\mathrm{x}}$ reaches its magnetization compensation composition at $\left(x_{\mathrm{MC}}\right) \sim 24$ at.\%. While the $M_{\mathrm{s}}$ is minimum and the coercive fields reach their maximum at magnetic compensation composition ${ }^{25,26}$. The angular momentum compensation composition $\left(x_{\mathrm{AMC}}\right)$ was determined using the following equations:

$$
\begin{aligned}
& M=M_{G d}+M_{F e C o}(2) \\
& A=A_{G d}+A_{F e C o}=\frac{M_{G d}}{\gamma_{G d}}+\frac{M_{F e C o}}{\gamma_{F e C o}}=\frac{M_{G d}}{\frac{g_{G d} \mu_{B}}{\hbar}}+\frac{M_{F e C o}}{\frac{g_{F e C o} \mu_{B}}{\hbar}}
\end{aligned}
$$

where $A_{\mathrm{i}}=M_{\mathrm{i}} / \gamma_{i}$, also $M_{\mathrm{i}}(\mathrm{i}=\mathrm{Gd}$ or $\mathrm{FeCo})$ is the magnetic moment and $\gamma_{i}=g_{\mathrm{i}} \mu_{\mathrm{B}} / \mathrm{h}$ is the corresponding g factor, $\mu_{\mathrm{B}}$ and $\mathrm{\hbar}$ is the Bohr magnetron and Planck constant, respectively. The $\mathrm{g}$ factor is $g_{\mathrm{Gd}} \approx 2.0$ and $g_{\mathrm{FeCo}} \approx 2.16^{27,24}$, hence, the $x_{\mathrm{AMC}}$ is estimated to be $x_{\mathrm{AMC}} \sim 23$, which is indicated in Fig. 2(b). Figure 2(c) shows the polar Kerr magneto-optical images of the $\mathrm{Pt} / \mathrm{Gd}_{\mathrm{x}}\left(\mathrm{Fe}_{88} \mathrm{Co}_{12}\right)_{100-\mathrm{x}} / \mathrm{SiN}$ wire (see supplementary Fig. 1 for details). First, a large external magnetic field along the out-of-plane is applied to saturate the magnetization and generate an up-down and/or down-up DW to initiate DW propagation from the large contact 
pad area. Then a pulse current is injected into a large contact pad. Therefore, the position of DW is displaced before and after applying a pulse current. Red and blue dotted lines show the initial and the final position of domain walls in the PMOKE image, respectively. The DW velocities were determined by dividing the DW displacement by the pulse duration, which is a general experimental method of the CIDWM in $\mathrm{Gd}_{\mathrm{x}}\left(\mathrm{Fe}_{88} \mathrm{Co}_{12}\right)_{100-\mathrm{x}}$ wires.

\section{Current induced domain wall motion}

Figure 3(a),(b) show the dependence of DW velocity as a function of current density for $\mathrm{Pt} / \mathrm{Gd}_{\mathrm{x}}\left(\mathrm{Fe}_{88} \mathrm{Co}_{12}\right)_{100-\mathrm{x}} / \mathrm{SiN}$ wires $(20<\mathrm{x}<28)$ measured with a pulse duration of 30 and $3 \mathrm{nsec}$, respectively. In our study, the DW moves along the direction of pulse current, which can be supported by the spin-orbit torque (SOT) in the heavy metal/ferrimagnet system ${ }^{28,29}$. Figure 3(a),(b) shows that the DW velocity increases by raising the $J$ for all $\operatorname{Gd}_{x}\left(\mathrm{Fe}_{88} \mathrm{Co}_{12}\right)_{100-\mathrm{x}}$ compositions. The maximum DW velocity $v_{\mathrm{DW}}=750 \mathrm{~m} / \mathrm{s}$ and $2000 \mathrm{~m} / \mathrm{s}$ are observed with a sample of $\mathrm{Gd}_{24}\left(\mathrm{Fe}_{88} \mathrm{Co}_{12}\right)_{76}$ for 30 and 3 nsec pulse duration, respectively. As a result, without applying an in-plane external magnetic field, the fastest DW velocity of $2000 \mathrm{~m} / \mathrm{s}$ has been obtained as far as we know in the reports. In addition, the domain wall velocity of both the up-down and down-up domain walls was the same $2000 \mathrm{~m} / \mathrm{s}$. In racetrack memory, it is necessary to shift recorded data patterns with the current without destroying them. Therefore, the results show that high-speed data transfer is possible as racetrack memory. On the other hand, the result ${ }^{22}$ of achieving $5700 \mathrm{~m} / \mathrm{sec}$ by accelerating only one side DW of the recorded magnetic domain using the in-plane external magnetic field is attracting attention, however, the DW on the other side decelerates significantly, in consequence, the method using the in- 
plane external magnetic field cannot be used practically, because it causes data destruction $22,3,2,30$.

Figure 3(c) exhibits the DW velocity as a function of Gd concentration under the condition with a constant current density of $J=1.7 \times 10^{11} \mathrm{~A} / \mathrm{m}^{2}$. It is seen that the DW velocity increases by increasing Gd content up to $\mathrm{x}=24$, and then $v_{\mathrm{DW}}$ decreases by increasing $\mathrm{Gd}$ content over $\mathrm{x}=25$. The maximum DW velocity $v_{\mathrm{DW}}=1500 \mathrm{~m} / \mathrm{s}(>20 \mathrm{Gbps})$ appears between $x_{\mathrm{MC}}$ and $x_{\mathrm{AMC}}$ point for $\mathrm{Gd}_{24} \mathrm{FeCo}_{76}$ with short pulse current 3 nsec.

Except for $\mathrm{Gd}_{24}\left(\mathrm{Fe}_{88} \mathrm{Co}_{12}\right)_{76}$, we observed a linear relationship between DW velocity with current density for all GdFeCo compositions (Fig. 3(a-b)). To clarify the $J$ dependence of DW velocity, we first determine the magnetic features of the $\mathrm{Gd}_{\mathrm{x}}\left(\mathrm{Fe}_{88} \mathrm{Co}_{12}\right)_{100-\mathrm{x}}$ wires using the hysteresis loops at various temperatures (as shown in the supplementary Fig. 2). From those results, the magnetic compensation temperature $\left(T_{\mathrm{MC}}\right)$ for each composition is obtained. Next, using the $T_{\mathrm{MC}}$, the angular momentum compensation temperature $T_{\mathrm{AMC}}$ was evaluated from Equations 1-4 of the supplement. As can be seen from Equation 1 of the supplement, the DW velocity becomes faster near the $T_{\mathrm{MC}}$. On the other hand, it is considered that the DW velocity increases even near the angular momentum compensation temperature $\left(T_{\mathrm{AMC}}\right)$. Figure 3(d) shows that the $T_{\mathrm{MC}}$ (green dots) and $T_{\mathrm{AMC}}$ (violet dote) are different for each $\mathrm{Gd}_{\mathrm{x}}\left(\mathrm{Fe}_{88} \mathrm{Co}_{12}\right)_{100-\mathrm{x}}$ composition. By increasing the Gd concentration the $T_{\mathrm{MC}}$ (green dots) and $T_{\text {AMC }}$ (violet dote) were shifted towards the higher temperature.

Since an electric current is applied to the magnetic wire at room temperature, it is desirable that $T_{\mathrm{AMC}}$ and $T_{\mathrm{MC}}$ are present near room temperature to achieve a high DW velocity. It can 
be considered that the reason why the DW velocity of $\mathrm{Gd}_{24}\left(\mathrm{Fe}_{88} \mathrm{Co}_{12}\right)_{76}$ is the fastest in Fig. 3(c) is that it meets this condition. Joule heat generation ${ }^{31,32}$ can be taken into account that, by applying a pulse current, nevertheless the longer the pulse width, the higher the Joule heat generation. When a pulse width is $3 \mathrm{nsec}$, the Joule heat generation is small, and the temperature of the magnetic wire is close to $T_{\mathrm{AMC}}$ as shown by the blue cross mark in Fig. 3(d). Therefore, it is seen that the DW velocity of the $\mathrm{Gd}_{24}\left(\mathrm{Fe}_{88} \mathrm{Co}_{12}\right)_{76}$ shows the highest in the GdFeCo in Fig. 3(c). On the other hand, when the pulse width is 30nsec, it is considered that the temperature of the magnetic wire has risen to the vicinity of the orange cross mark in Fig. 3(d). The result of the temperature simulation is described in Fig. 4(b). Therefore, as a result of the current density dependence of the DW velocity with a pulse width of $30 \mathrm{nsec}$ in Fig. 3(a), the temperature of the magnetic wire is near $T_{\mathrm{AMC}}$, so the DW velocity becomes faster. However, when the current is further increased, the temperature exceeds the $T_{\text {AMC, }}$ so the DW velocity slows down. Thus, only this 30nsec pulse injection result of $\mathrm{Gd}_{24}\left(\mathrm{Fe}_{88} \mathrm{Co}_{12}\right)_{76}$ does not show a straight line due to this effect.

By applying excessive current density, multi-domains can be created easily for the samples which are far from the magnetic compensation composition point $x_{\mathrm{MC}}$. For these samples, the perpendicular anisotropy $K_{\text {eff }}$ becomes smaller than that of samples near the $x_{\mathrm{MC}} \mathrm{C}^{33,34}$. Thus, it is taken into account that a short pulse current is required to avoid multidomains at high current density $J$. As a result, to apply larger current densities $(J)$ into the Pt/ $\mathrm{Gd}_{\mathrm{x}}\left(\mathrm{Fe}_{88} \mathrm{Co}_{12}\right)_{100-\mathrm{x}}$ wires and determine the faster DW motion in magnetic wires, we compared the CIDWM using single voltage pulses with a pulse duration of 30nsec and 3nsec for all $\mathrm{Gd}_{\mathrm{x}}\left(\mathrm{Fe}_{88} \mathrm{Co}_{12}\right)_{100-\mathrm{x}}$ composition, respectively. A wide range of current densities can be 
injected into the samples among $x_{\mathrm{MC}}$ and $x_{\mathrm{AMC}}$ using a short pulse current. Accordingly, the fast DW motion at low current density can be realized in $\mathrm{Gd}_{\mathrm{x}}\left(\mathrm{Fe}_{88} \mathrm{Co}_{12}\right)_{100-\mathrm{x}}$ wires without using an external magnetic field.

\section{Thermal stability and fast domain wall motion without external magnetic field}

Generally, to attain even fast DW velocity, larger current densities are needed. Figure 4(a) shows the DW velocity as a function of pulse duration for the $\mathrm{Gd}_{25}\left(\mathrm{Fe}_{88} \mathrm{Co}_{12}\right)_{75}$ sample. Despite applying the same current density $\left(J=1.7 \times 10^{11} \mathrm{~A} / \mathrm{m}^{2}\right)$, the DW velocity becomes faster as the pulse duration becomes shorter. As discussed previously, in ferrimagnetic materials the temperature can affect their magnetic properties ${ }^{31}$.

Increasing the input current density also increases the domain wall velocity, as shown in Fig. 3(a),(b). When the pulse width is fixed at 3nsec, and the number of pulses is increased, the DW displacement distance is ideally proportional to the number of pulses, as shown in the inset of Fig. 4(a). Similarly, fixing the current density and increasing the pulse width should increase the domain wall velocity. However, when the current density was fixed $(J$ $=1.7 \times 10^{11} \mathrm{~A} / \mathrm{m}^{2}$ ) and the pulse width was increased as shown in Fig.4(a), the domain wall velocity became slower, contrary to the expectation. Hence, this expectation can be explained due to the Joule heating effect in the GdFeCo wires. As shown in Fig. 4(b) the effect of Joule heating on the DW velocity can be clarified as follows: When the applied current flows through the magnetic wire, the temperature in the GdFeCo layer rises, which depends on pulse duration. However, an increase in the sample temperature with higher current density will drive the sample away from the compensation region, then the DW velocity decreases. 
The Joule heating effect decreases the DW velocity for the duration of the large pulses current, which is consistent with the previous reports ${ }^{21,3}$.

Our experimental data shows that fast and stable DW motion occurs in short pulse current. Correlated with these large current densities is a substantial amount of Joule heating that enhances the temperature of the samples. Therefore, it is a crucial issue to recognize that how strongly the temperature can influence the physical properties of ferrimagnetic wires. To determine the temperature change induced by the pulse duration, we performed a numerical simulation based on the heat diffusion in wires (as shown in Supplementary Figs. 4-5). Figure 4(b) exhibits the temperature profile after the application of a current density of $J=1.7 \times 10^{11}$ $\mathrm{A} / \mathrm{m}^{2}$ at the different pulse duration as a function of position $x$. Figure $4(\mathrm{~b})$ presents that the temperature of the samples increased at the center of the wire for both $30 \mathrm{nsec}$ and $3 \mathrm{nsec}$ pulse duration. It is seen that the high-temperature gradient occurs between the edge part and center part of the GdFeCo wire when the 30nsec pulse current is injected into the sample. Contrarily, the $3 \mathrm{nsec}$ pulse duration shows a broad peak (small temperature gradient) at the center of the wire. Accordingly, the temperature gradient increases linearly and causes decreases in the DW velocity with a negative linear relationship with the sample temperature (as shown in the supplementary Fig. 6). Therefore, it is clearly observed that the DW propagation is more uniform and stable when a short pulse current of $3 \mathrm{nsec}$ is injected into the sample, which agrees with experimental results in Figure 4(a).

Figure 4(c) shows the DW velocity of the $\mathrm{Gd}_{24}\left(\mathrm{Fe}_{88} \mathrm{Co}_{12}\right)_{76}$ as a function of operating temperature for pulse current of $30 \mathrm{nsec}$ and $3 \mathrm{nsec}$, respectively. DW speed obtained stable 
between the temperature range of R.T. $<T_{\mathrm{op}}<70^{\circ} \mathrm{C}$ for $\mathrm{Gd}_{24} \mathrm{FeCo}_{76}$ when pulse current of $30 \mathrm{nsec}$ and $3 \mathrm{nsec}$ is injected into the wire. Especially for a short pulse current of $3 \mathrm{nsec}$, the DW velocity remains relatively constant, over $1200 \mathrm{~m} / \mathrm{s}$ between room temperature and $70{ }^{\circ} \mathrm{C}$, which is suitable for the racetrack memories (see supplementary Fig. 3 for details). As a result, a wide temperature range with a DW velocity of $1200 \mathrm{~m} / \mathrm{s}$ has been obtained in $\mathrm{Gd}_{24}\left(\mathrm{Fe}_{88} \mathrm{Co}_{12}\right)_{76}$ for a short pulse current which is more stable in comparison with the previous reports ${ }^{21,3,35}$.

\section{The physical mechanism of fast domain wall motion in GdFeCo}

The experimental realization of this proposal is shown in Fig. 5(a-d) which the DW velocity is a function of $\mathrm{Tb}$ and $\mathrm{Gd}$ concentration. So far, we have reported many papers on the current driven DW motion of TbCo magnetic wires, but the DW velocity was as slow as $100 \mathrm{~m} / \mathrm{sec}$ or less even if the composition and structure were changed ${ }^{1,2,36}$. Previous studies clarified that the fast DW motion appears at the angular momentum compensation point, which is consistent with the results given by Saima et $\mathrm{al}^{2}$. We first extracted the TbCo DW velocity as a function of $\mathrm{Tb}$ concentration in Fig. 5(c). Saima et al. have shown that the highest DW speed attains a maximum at around $\mathrm{x} \approx 0.21-26$. Fig. $5(\mathrm{c})$ presents that the $x_{\mathrm{AMC}}$ is far from the $x_{\mathrm{MC}}$ due to the different $\mathrm{g}$ values for $\mathrm{Tb} \sim 1.5$ and $\mathrm{Co} \sim 2.22$ atoms. It is seen that to reach the maximum DW velocity of TbCo sample has a narrow margin of $\mathrm{Tb}$ composition between $x_{\mathrm{AMC}}$ and $x_{\mathrm{MC}}$. Thus, it is difficult to accelerate the DW velocity of the TbCo wires. Figure 5(d) shows the DW velocity dependence of Gd composition and presents that DW velocity reaches its maximum between $x_{\mathrm{AMC}}$ and $x_{\mathrm{MC}}$. Hence, The GdFeCo sample has a wide 
margin of Gd composition between $x_{\mathrm{MC}}$ and $x_{\mathrm{AMC}}$ for the reason that the $\mathrm{g}$ values of $\mathrm{Gd}$ and $\mathrm{Fe}_{88} \mathrm{Co}_{12}$ are close to each other. These results show that only the angular momentum compensation point is insufficient to obtain the fast DW motion in compensated ferrimagnet. $\mathrm{We}$, therefore, propose that bringing the $\mathrm{g}$ values of RE and TM near to each other is essential to design a fast DW motion.

In order to realize a fast data rate of $20 \mathrm{Gbps}$, both low current density $J$ and fast DW motion are required. Therefore, large DW mobility $v_{\mathrm{DW}} / J$ is an important parameter. To compare our work with previous reports, it is clear that the DW mobility in the GdFeCo sample is much higher than that of other FM and FIM materials, which is shown in Fig. 5(e) $28,29,2,8,21$. The DW mobility for 30 and 3 nsec pulse width is $33 \times 10^{-10} \mathrm{~m}^{3} /$ As and $88 \times 10^{-10}$ $\mathrm{m}^{3} /$ As. These results open a new window for technology to design a new type of racetrack memory with low power consumption and high-velocity thermal stability.

In summary, we achieve a wide temperature range and fast domain wall motion $\left(v_{\mathrm{DW}}>1200 \mathrm{~m} / \mathrm{s}\right)$ between magnetic and angular momentum compensation points without an external magnetic field. We have experimentally demonstrated and theoretically explained that a short pulse current of $3 \mathrm{nsec}$ is more stable and faster than a long pulse width of $30 \mathrm{nsec}$. Our work implies that bringing the $g$ values of RE and TM close to each other is a fundamental parameter for designing fast and stable racetrack memory for $5 \mathrm{G}$ applications. These results could be a clue to realize the physical mechanism of DW motion in amorphous ferrimagnetic alloys, and therefore DW mobility is remarkably enhanced more than 10-times, which will invigorate research towards the development of memory applications. 


\section{Methods}

Sample preparations. A series of $\mathrm{Pt}(5) / \mathrm{Gd}_{\mathrm{x}}\left(\mathrm{Fe}_{88} \mathrm{Co}_{12}\right)_{100-\mathrm{x}}(20) / \mathrm{SiN}(10)$ films were deposited by dc magnetron sputtering under an Ar pressure of $0.2 \mathrm{~Pa}$ after the chamber was evacuated to a base pressure of about $2 \times 10^{-8}$ torr. Here the $5 \mathrm{~nm}$ thick Pt under layer performs as a spin current source induced by spin Hall effect (SHE), which provides a spin-orbit torque (SOT). A $10 \mathrm{~nm}$ thick $\mathrm{SiN}$ layer is fabricated on the top of the $\mathrm{Gd}_{\mathrm{x}} \mathrm{FeCo}_{100-\mathrm{x}}$ layer to prevent oxidization. The GdFeCo alloy layers were deposited by co-sputtering FeCo and Gd targets at room temperature. The magnetic wires ( $3 \mu \mathrm{m}$ wide and $125 \mu \mathrm{m}$ long) and Hall crosses were micro-fabricated by an Electron-beam lithography (EBL) system and a lift-off method. When data with the minimum bit length of $60 \mathrm{~nm}$ is recorded on half of a $125 \mu \mathrm{m}$ magnetic wire, 1024-bit data can be recorded as a high-speed, ultra-power-saving resister. Currently, registers that combine many SRAMs are used, and our results suggest that the power consumption and cost of the register can be significantly reduced.

Magnetic measurements. The Gd and FeCo composition can be controlled by changing the Gd and FeCo sputtering power. The composition of the films was measured by energy dispersive X-ray analysis (EDX). The magnetic properties were measured at room temperature by the Polar-magneto-optical Kerr effect (PMOKE) and vibrating sample magnetometer (VSM). We measured the domain wall velocity in the GdFeCo wires by applying single voltage pulses (Picosecond) and then observed the DW motion by using a Kerr microscope. The velocity of the domain wall was determined by dividing the change in 
the position of the domain wall (as viewed by PMOKE) by the duration of the current pulse (Fig. 2(c)). Note that all measurements are performed at room temperature. 


\section{References}

1. Bang, D., Van Thach, P. \& Awano, H. Current-induced domain wall motion in antiferromagnetically coupled structures: Fundamentals and applications. J. Sci. Adv. Mater. Devices 3, 389-398 (2018).

2. Siddiqui, S. A., Han, J., Finley, J. T., Ross, C. A. \& Liu, L. Current-Induced Domain Wall Motion in a Compensated Ferrimagnet. Phys. Rev. Lett. 121, 57701 (2018).

3. Kim, K. J. et al. Fast domain wall motion in the vicinity of the angular momentum compensation temperature of ferrimagnets. Nat. Mater. 16, 1187-1192 (2017).

4. Parkin, S. S. P., Hayashi, M. \& Thomas, L. Magnetic domain-wall racetrack memory. Science (80-. ). 320, 190-194 (2008).

5. Komine, T., Takahashi, K., Ooba, A. \& Sugita, R. Reduction of intrinsic critical current density for current-induced domain wall motion by using a ferrimagnetic nanowire with perpendicular magnetic anisotropy. J. Appl. Phys. 109, 1-4 (2011).

6. Hiroyuki Awano, Susumu Imai, Masaki Sekine, Manabu Tani, Norio Ohta, Kenichiro Mitani, Nobuyuki Takagi, Hitoshi Noguchi, and M. K. $20 \mathrm{~nm}$ Domain Expansion Readout by Magnetic Amplifying MO System (MAMMOS). IEEE Trans. Magn. 36, 2261-2265 (2000).

7. Fukami, S., Ieda, J. \& Ohno, H. Thermal stability of a magnetic domain wall in nanowires. Phys. Rev. B - Condens. Matter Mater. Phys. 91, 1-7 (2015).

8. Yang, S. H., Ryu, K. S. \& Parkin, S. Domain-wall velocities of up to $750 \mathrm{~m} \mathrm{~s}-1$ driven by exchange-coupling torque in synthetic antiferromagnets. Nat. Nanotechnol. 10, 221-226 (2015).

9. Jungwirth, T., Marti, X., Wadley, P. \& Wunderlich, J. Antiferromagnetic spintronics. Nat. Nanotechnol. 11, 231-241 (2016).

10. Büttner, F., Lemesh, I. \& Beach, G. S. D. Full phase diagram of isolated skyrmions in a ferromagnet. arXiv 1-12 (2017) doi:10.1038/s41598-018-22242-8.

11. Cheng, R., Xiao, D. \& Brataas, A. Terahertz Antiferromagnetic Spin Hall NanoOscillator. Phys. Rev. Lett. 116, 1-5 (2016).

12. Olejnik, K. et al. THz electrical writing speed in an antiferromagnetic memory. arXiv 1-9 (2017).

13. Cheng, R. \& Niu, Q. Dynamics of antiferromagnets driven by spin current. Phys. Rev. B - Condens. Matter Mater. Phys. 89, 1-5 (2014).

14. Kim, S. K., Tchernyshyov, O. \& Tserkovnyak, Y. Thermophoresis of an antiferromagnetic soliton. Phys. Rev. B - Condens. Matter Mater. Phys. 92, 1-5 
(2015).

15. Zhang, X., Zhou, Y. \& Ezawa, M. Antiferromagnetic Skyrmion: Stability, Creation and Manipulation. Sci. Rep. 6, 1-8 (2016).

16. Kaiser, C., Panchula, A. F. \& Parkin, S. S. P. Finite tunneling spin polarization at the compensation point of rare-earth-metal-transition-metal alloys. Phys. Rev. Lett. 95, $1-4$ (2005).

17. Jeong, J. et al. Termination layer compensated tunnelling magnetoresistance in ferrimagnetic Heusler compounds with high perpendicular magnetic anisotropy. Nat. Commun. 7, 1-8 (2016).

18. Yoshimura, Y. et al. Current-induced domain wall motion in perpendicularly magnetized $\mathrm{Co} / \mathrm{Ni}$ nanowire under in-plane magnetic fields. Appl. Phys. Express 5, 21-24 (2012).

19. Yamaguchi, A. et al. Real-Space Observation of Current-Driven Domain Wall Motion in Submicron Magnetic Wires. Phys. Rev. Lett. 92, 1-4 (2004).

20. Finley, J. \& Liu, L. Spintronics with compensated ferrimagnets. Appl. Phys. Lett. 116, (2020).

21. Caretta, L. et al. Fast current-driven domain walls and small skyrmions in a compensated ferrimagnet. Nat. Nanotechnol. 13, 1154-1160 (2018).

22. Cai, K. et al. Ultrafast and energy-efficient spin-orbit torque switching in compensated ferrimagnets. Nat. Electron. 3, 37-42 (2020).

23. Hirata, Y. et al. Correlation between compensation temperatures of magnetization and angular momentum in GdFeCo ferrimagnets. Phys. Rev. B 97, 1-6 (2018).

24. Scott, G. G. Review of gyromagnetic ratio experiments. Rev. Mod. Phys. 34, 102109 (1962).

25. Kato, T. et al. Compositional dependence of g-factor and damping constant of GdFeCo amorphous alloy films. IEEE Trans. Magn. 44, 3380-3383 (2008).

26. Kawakami, K., Kato, T., Oshima, D. \& Iwata, S. Spin orbit torques in ferrimagnetic GdFeCo with various compositions. Jpn. J. Appl. Phys. 59, (2020).

27. Gangulee \& R. C. Taylor and A. Magnetic anisotropy in evaporated amorphous films of the ternary system Gdx(Fe1-yCoy)1-x. J. Appl. Phys. 358, (1976).

28. Emori, S., Bauer, U., Ahn, S. M., Martinez, E. \& Beach, G. S. D. Current-driven dynamics of chiral ferromagnetic domain walls. Nat. Mater. 12, 611-616 (2013).

29. Ryu, K. S., Thomas, L., Yang, S. H. \& Parkin, S. Chiral spin torque at magnetic domain walls. Nat. Nanotechnol. 8, 527-533 (2013). 
30. Bläsing, R. et al. Exchange coupling torque in ferrimagnetic $\mathrm{Co} / \mathrm{Gd}$ bilayer maximized near angular momentum compensation temperature. Nat. Commun. 9, (2018).

31. Fangohr, H., Chernyshenko, D. S., Franchin, M., Fischbacher, T. \& Meier, G. Joule heating in nanowires. Phys. Rev. B - Condens. Matter Mater. Phys. 84, 1-12 (2011).

32. You, C. Y., Sung, I. M. \& Joe, B. K. Analytic expression for the temperature of the current-heated nanowire for the current-induced domain wall motion. Appl. Phys. Lett. 89, (2006).

33. Hansen, P., Klahn, S., Clausen, C., Much, G. \& Witter, K. Magnetic and magnetooptical properties of rare-earth transition-metal alloys containing Dy, Ho, Fe, Co. J. Appl. Phys. 69, 3194-3207 (1991).

34. Torrejon, J. et al. Interface control of the magnetic chirality in $\mathrm{CoFeB} / \mathrm{MgO}$ heterostructures with heavy-metal underlayers. Nat. Commun. 5, 4-11 (2014).

35. Okuno, T. et al. Spin-transfer torques for domain wall motion in antiferromagnetically coupled ferrimagnets. Nat. Electron. 2, 389-393 (2019).

36. Bang, D. \& Awano, H. Domain wall motion in $\mathrm{Tb} / \mathrm{Co}$ multilayer wires with a large domain wall depinning field. J. Appl. Phys. 115, 7-10 (2014). 


\section{Acknowledgments}

This work was supported by the JSPS KAKENHI (grants 20H02185, 21K14202)

\section{Author contributions}

S.R. and H.A. planned the project. S.R. deposited films, fabricated devices, and performed the transport measurements. S.K. performed a theoretical analysis. S.R., S.S., S.K., P.V.T, K.T., and H.A. analyzed the data. All authors discussed the results and commented on the manuscript. S.R. wrote the manuscript. H.A. and S.R. initiated the idea and led the project.

\section{Competing interests}

The authors declare no competing interests.

\section{Additional information}

Supplementary Information accompanies this paper at http://www.nature.com/nature

Reprints and permission information is available online at http://npg.nature.com/

Reprintsand permissions/

Correspondence and requests for materials should be addressed to S.R. (ina.ranjbar@toyota-ti.ac.jp) or H.A. (awano@toyota-ti.ac.jp). 


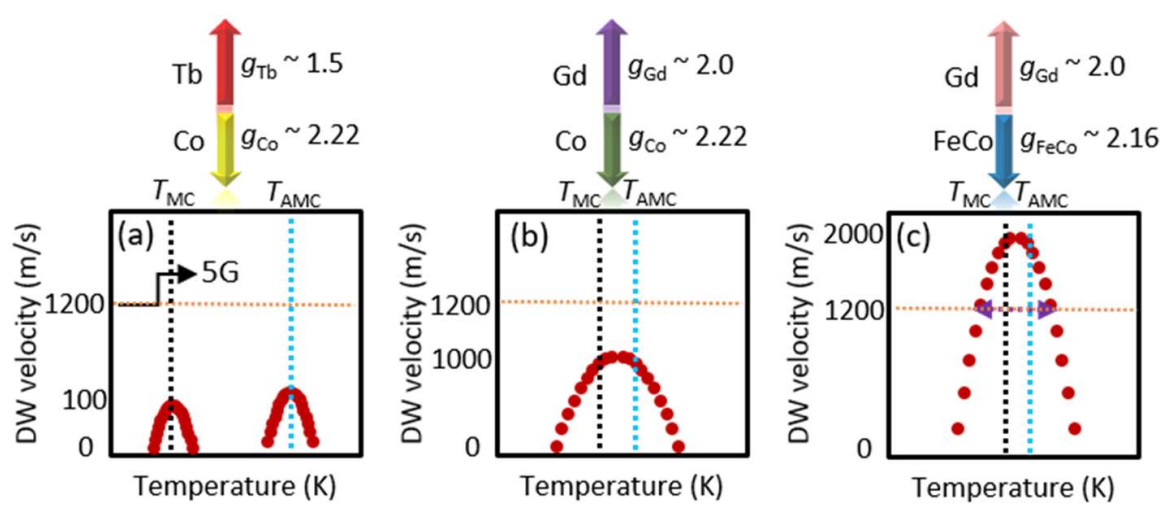

Figure 1. (a-c) Schematic illustration of the concept of DW motion in ferrimagnetic compared to our results.

Table 1. Compare of our work ( $\mathrm{GdFeCo})$ with other studies (TbCo and $\mathrm{GdCo}$ ).

\begin{tabular}{|c|c|c|c|}
\hline Parameters & $\mathrm{TbCo}^{29}$ & $\mathrm{GdCo}^{1}$ & GdFeCo (our results)* \\
\hline $\begin{array}{l}\text { DW speed }(\mathrm{m} / \mathrm{s}) \text { (room } \\
\text { temperature) }\end{array}$ & 75 & 850 & $1500^{*}$ \\
\hline $\mathrm{J}\left(\mathrm{A} / \mathrm{m}^{2}\right)$ & $3.0 \times 10^{11}$ & $20.0 \times 10^{11}$ & $1.7 \times 10^{11^{*}}$ \\
\hline DW mobility & 5 & 6.5 & $88^{*}$ \\
\hline $\begin{array}{l}\text { Thermal stability } \\
\left(v_{D w}=1200 \mathrm{~m} / \mathrm{s}\right)\end{array}$ & No report & Not stable & stable* \\
\hline$g(\operatorname{Re})^{23-25}$ & $\mathrm{~g}_{\mathrm{Tb}} \sim 1.5$ & $\mathrm{~g}_{\mathrm{Gd}} \sim 2.0$ & $\mathrm{~g}_{G d} \sim 2.0$ \\
\hline$g(T M)^{23-25}$ & $\mathrm{~g}_{\mathrm{co}} \sim 2.22$ & $\mathrm{~g}_{\mathrm{co}} \sim 2.22$ & $\begin{array}{l}\mathrm{g}_{\mathrm{Fe} 88 \mathrm{Co} 12} \sim 2.16^{*} \\
\left(\mathrm{~g}_{\mathrm{Fe}} \sim 2.15\right. \\
\left.\mathrm{g}_{\mathrm{Co}} \sim 2.22\right)\end{array}$ \\
\hline
\end{tabular}



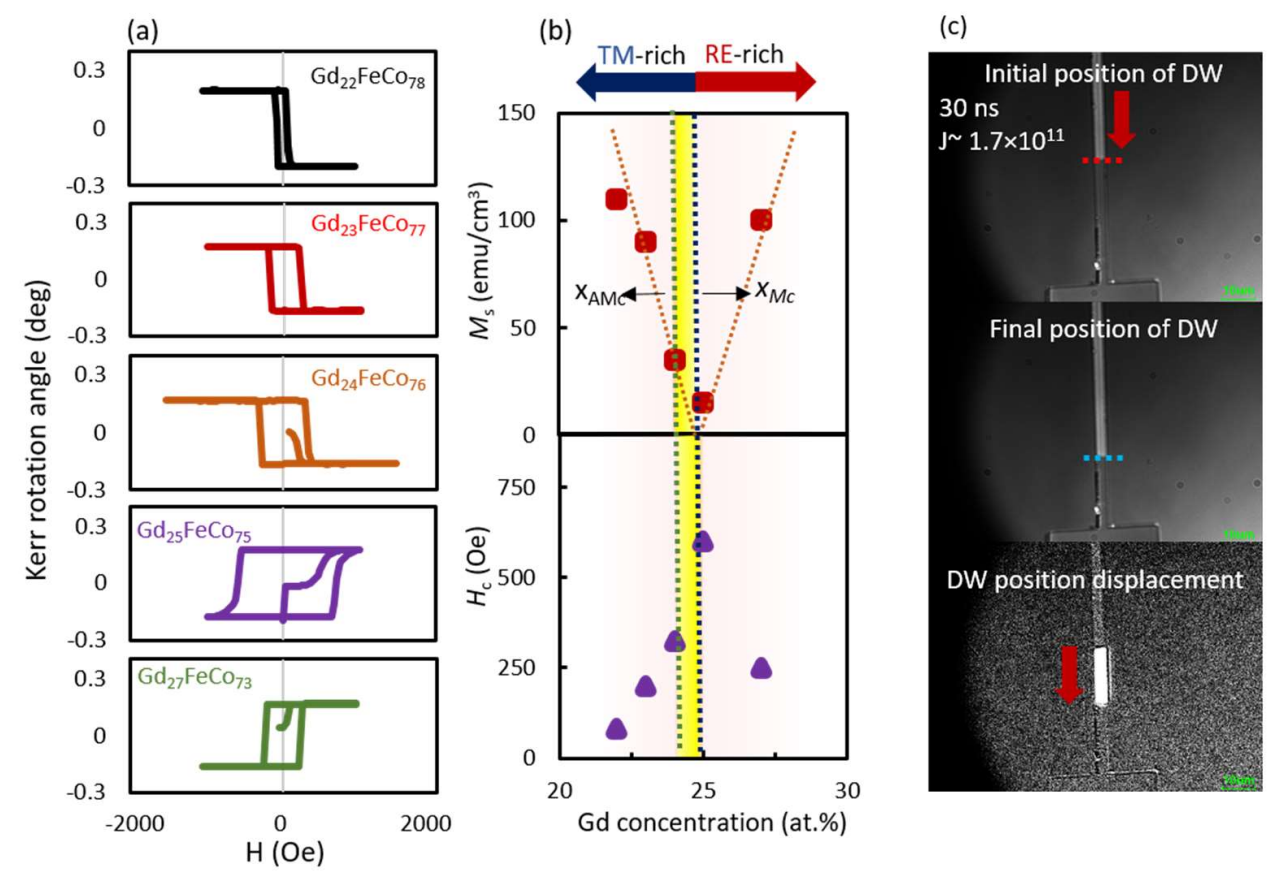

Figure 2. (a) MOKE measurements as a function of perpendicular magnetic field for $\mathrm{Gd}_{\mathrm{x}} \mathrm{FeCo}_{100-\mathrm{x}}$ films at room temperature. (b) saturation magnetization and coercivity as a function of $\mathrm{Gd}$ composition. (c) configuration of domain wall motion in $\mathrm{Gd}_{\mathrm{x}} \mathrm{FeCo}_{100-\mathrm{x}}$ wire with consecutive current pulses. 

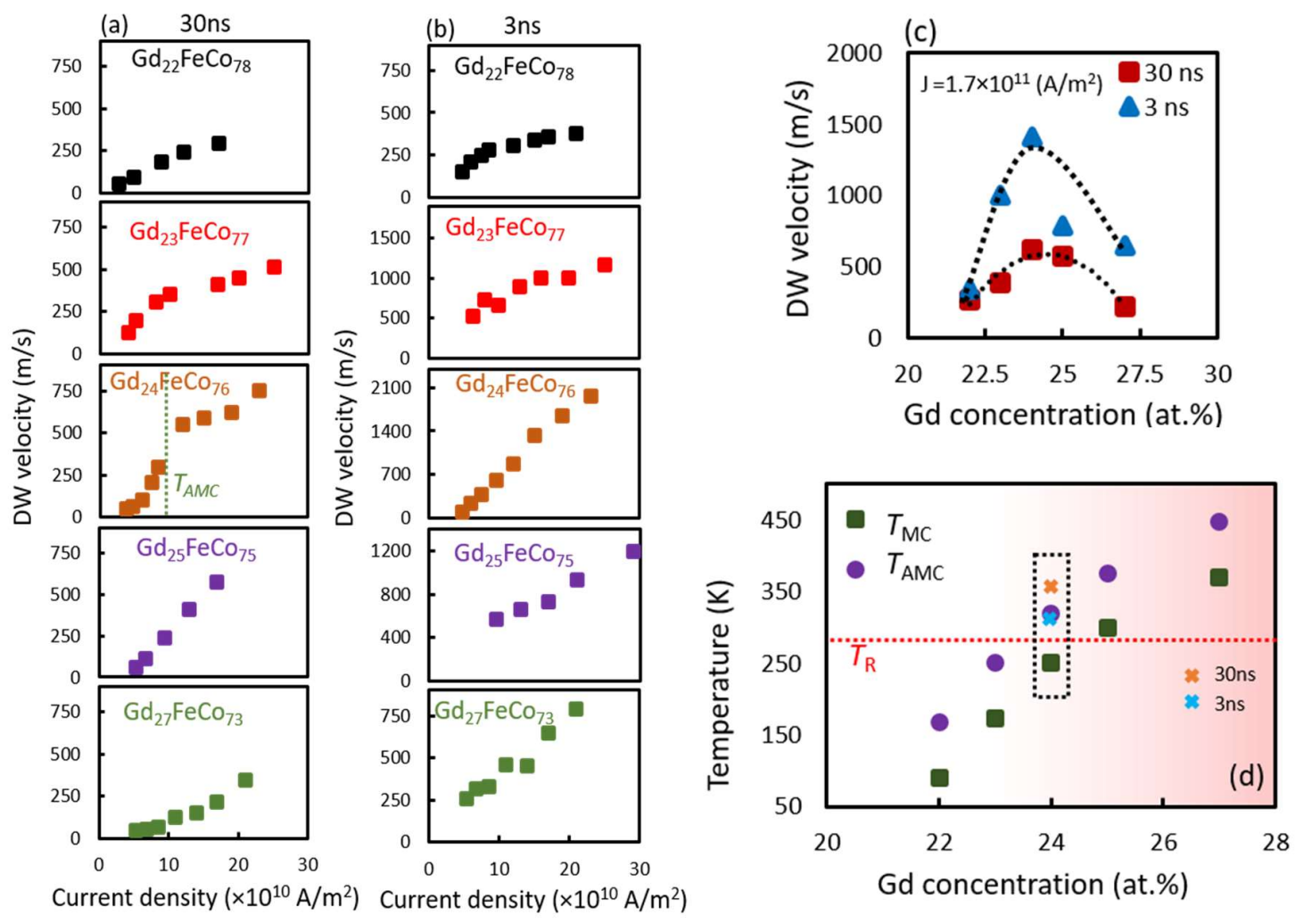

Figure 3. (a-b) Domain wall velocity as a function of current density with pulse duration $30 \mathrm{~ns}$ for $\mathrm{Gd}_{\mathrm{x}} \mathrm{FeCo}_{100-\mathrm{x}} / \mathrm{SiN}$ wires $(20<\mathrm{x}<28)$, (c) Domain wall velocity as a function of current density with pulse duration $30,3 \mathrm{nsec}$ for $\mathrm{Gd}_{\mathrm{x}} \mathrm{FeCo}_{100-\mathrm{x}} / \mathrm{SiN}$ wires $(20<\mathrm{x}<28)(\mathrm{d})$ summary of $T_{\mathrm{M}}$ and $T_{\mathrm{AM}}$ as a function of $\mathrm{Gd}$ concentration. For $\mathrm{Gd}_{24} \mathrm{FeCo}_{76}$ sample, the $T_{\mathrm{AMC}}$ is $320 \mathrm{~K}$ 

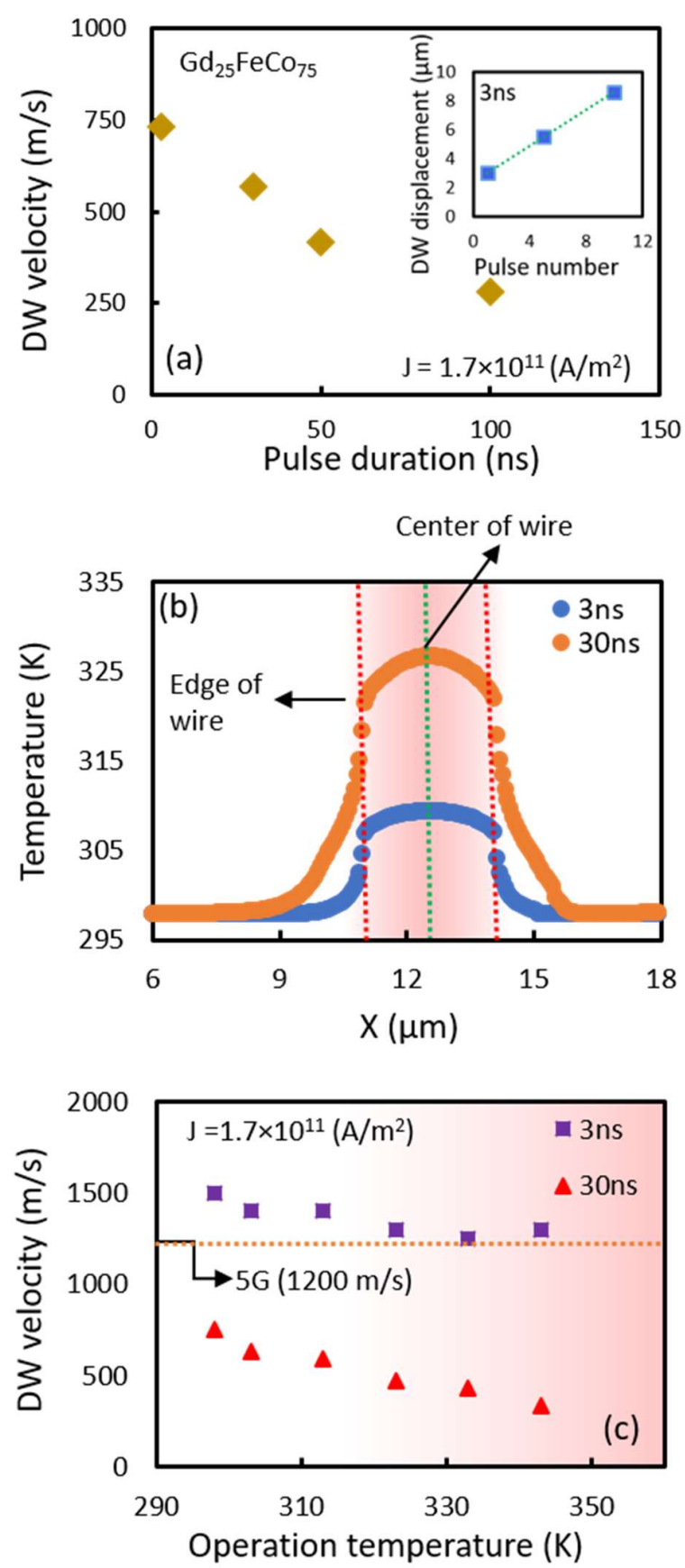

Figure 4. (a) the DW velocity as a function of pulse duration and the inset shows DW displacement as a function of pulse number (b) temperature profile after $t=30$ and $3 \mathrm{~ns}$ along the length of the wire on a silicon substrate (c) effect of operation temperature on DW speed for different pulse durations. 

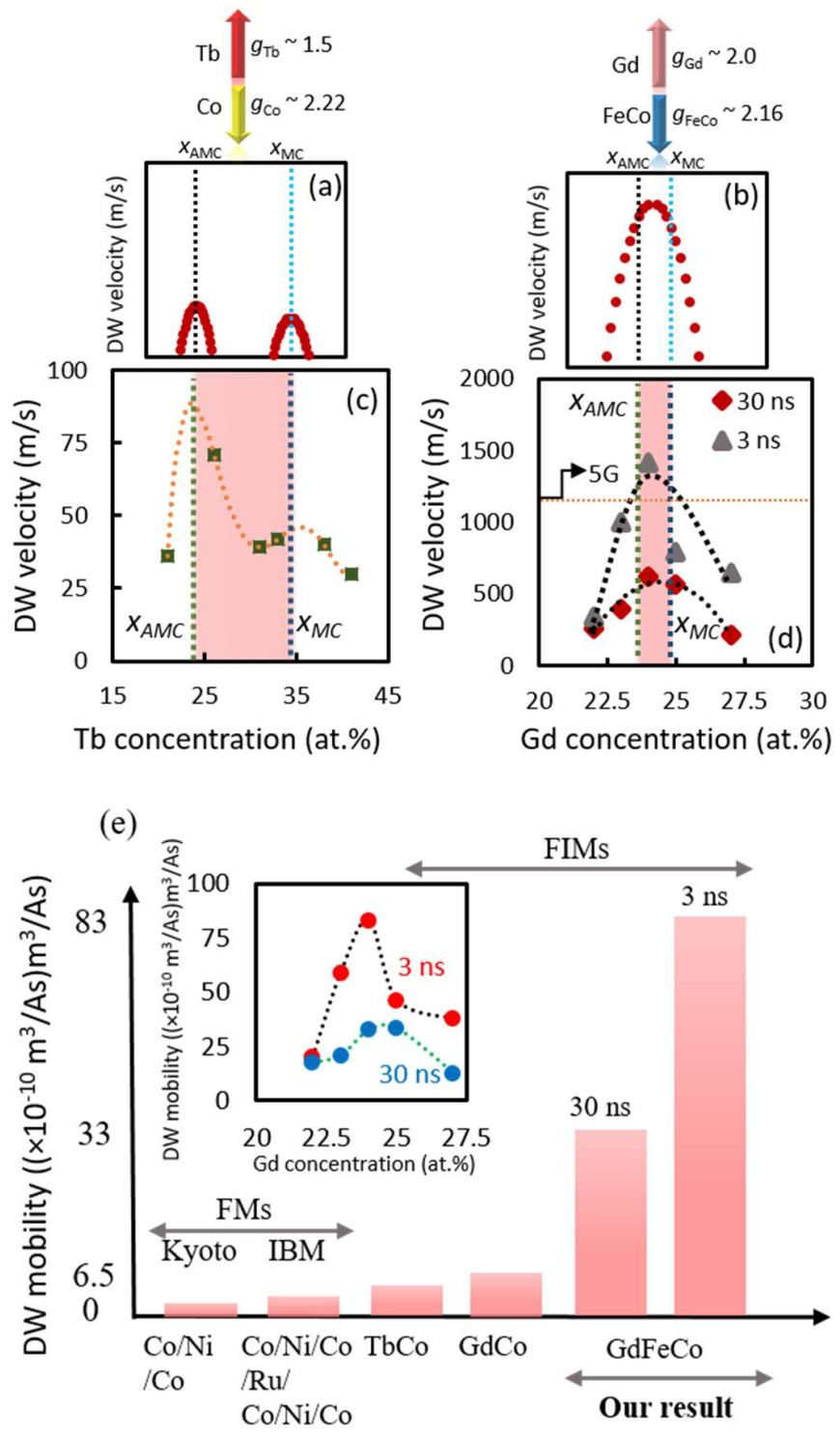

Figure 5. (a-b) schematic margin of TbCo and GdFeCo between angular momentum compensation composition and magnetic compensation composition (c-d) comparing $\mathrm{TbCo}^{29}$ with our GdFeCo samples (e) comparison of DW mobility in our study with other reports. 
Figures

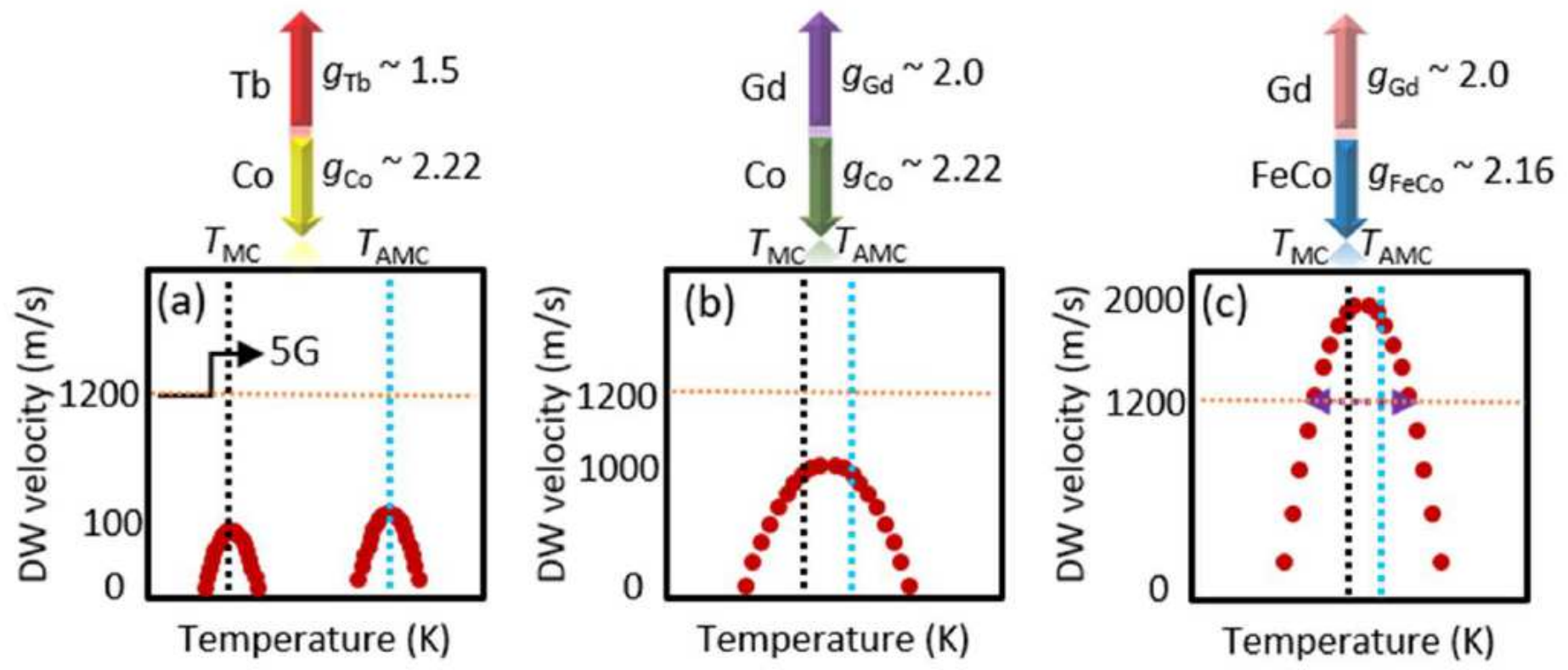

Figure 1

(a-c) Schematic illustration of the concept of DW motion in ferrimagnetic compared to our results. 
(a)

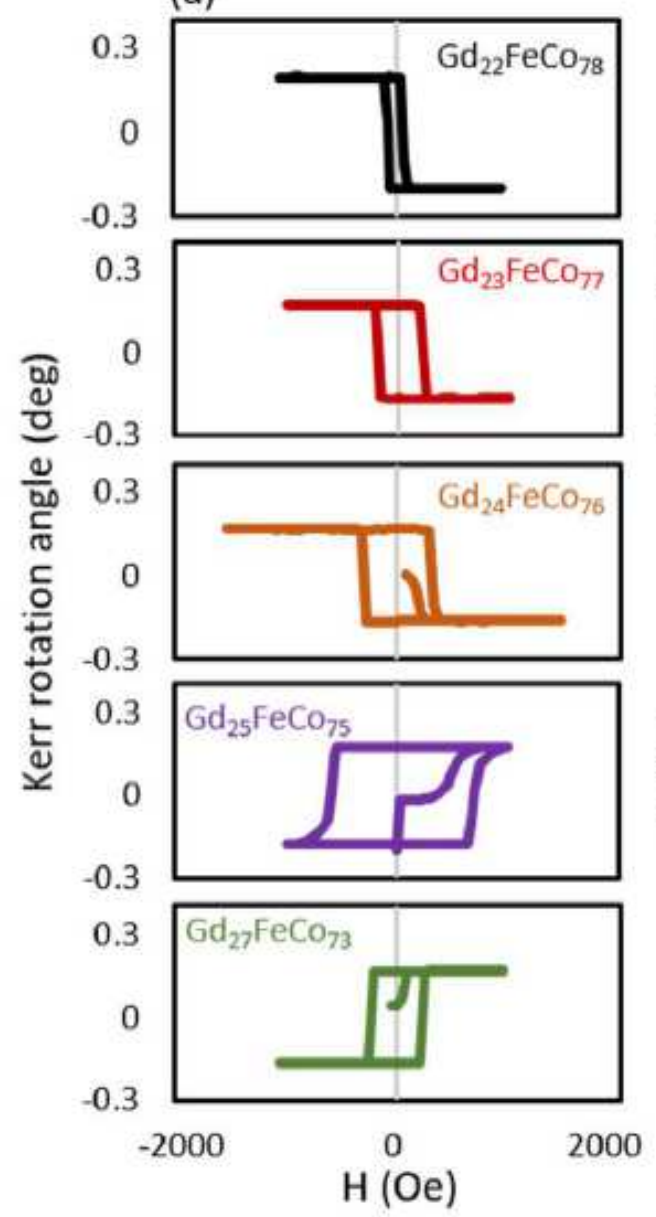

(b)

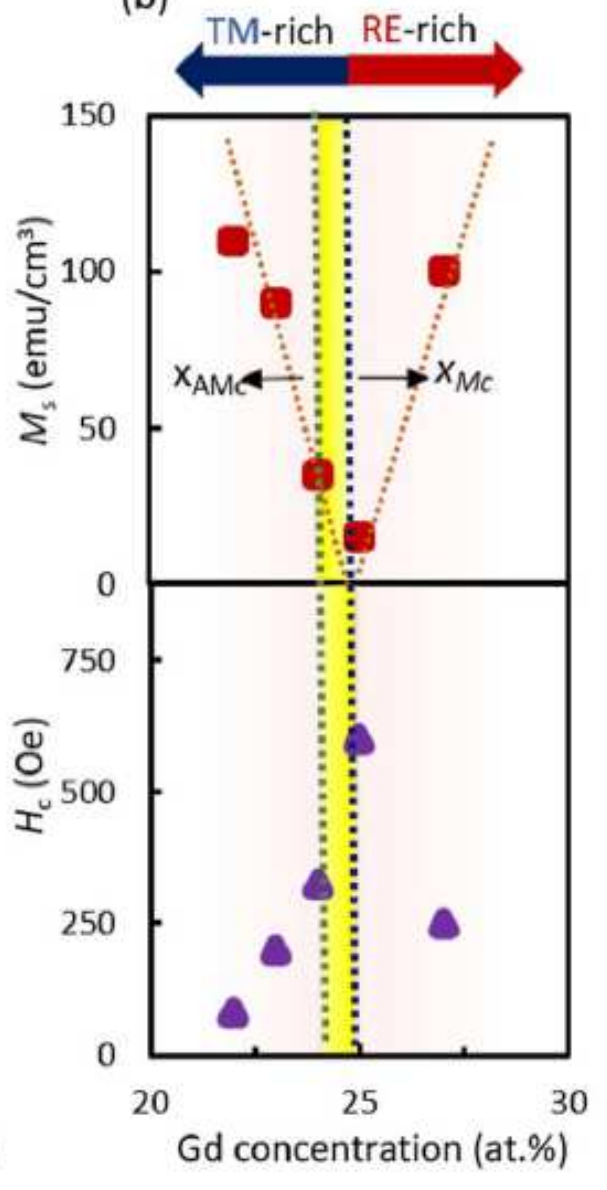

(c)

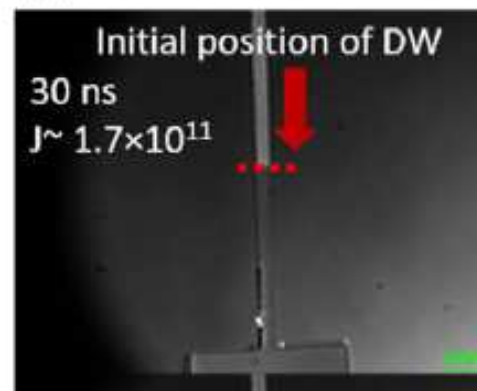

Final position of DW

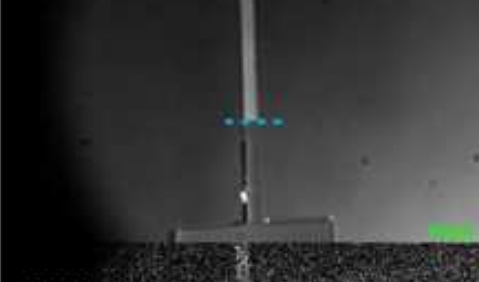

DW position displacement

\section{Figure 2}

(a) MOKE measurements as a function of perpendicular magnetic field for GdxFeCo100-x films at room temperature. (b) saturation magnetization and coercivity as a function of $\mathrm{Gd}$ composition. (c) configuration of domain wall motion in $\mathrm{GdxFeCo100-x}$ wire with consecutive current pulses. 

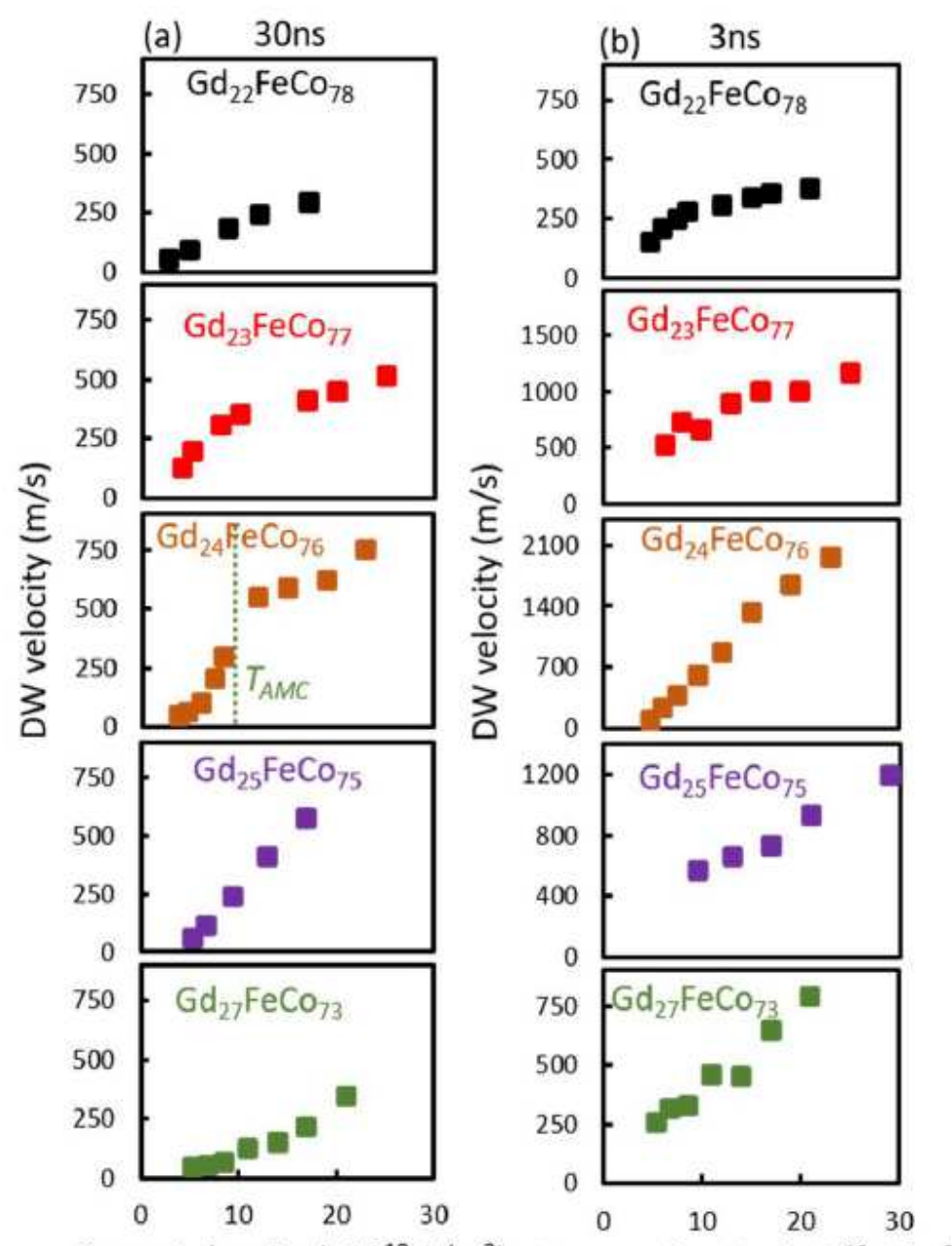

Current density $\left(\times 10^{10} \mathrm{~A} / \mathrm{m}^{2}\right)$ Current density $\left(\times 10^{10} \mathrm{~A} / \mathrm{m}^{2}\right)$
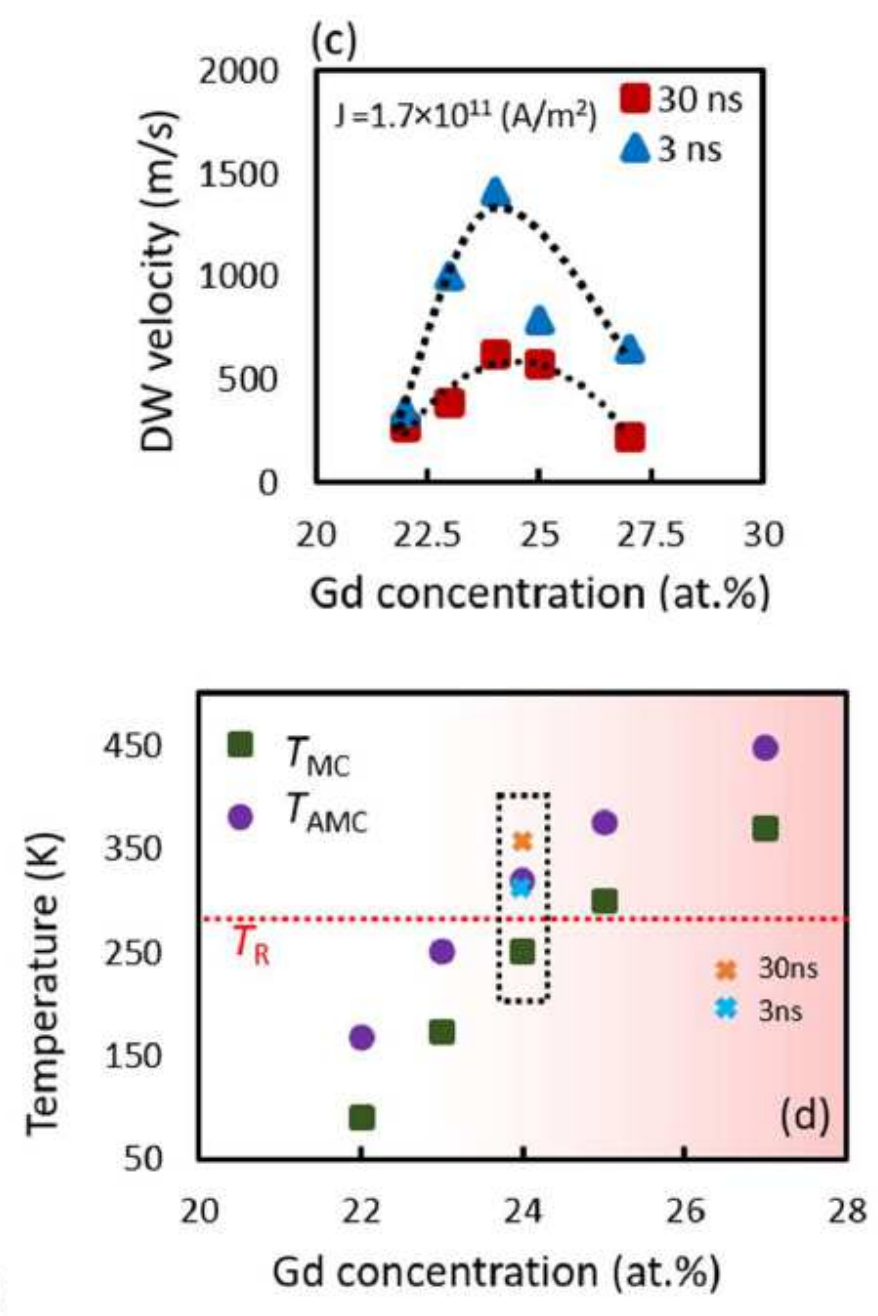

Figure 3

(a-b) Domain wall velocity as a function of current density with pulse duration 30 ns for GdxFeCo100-x /SiN wires (20 

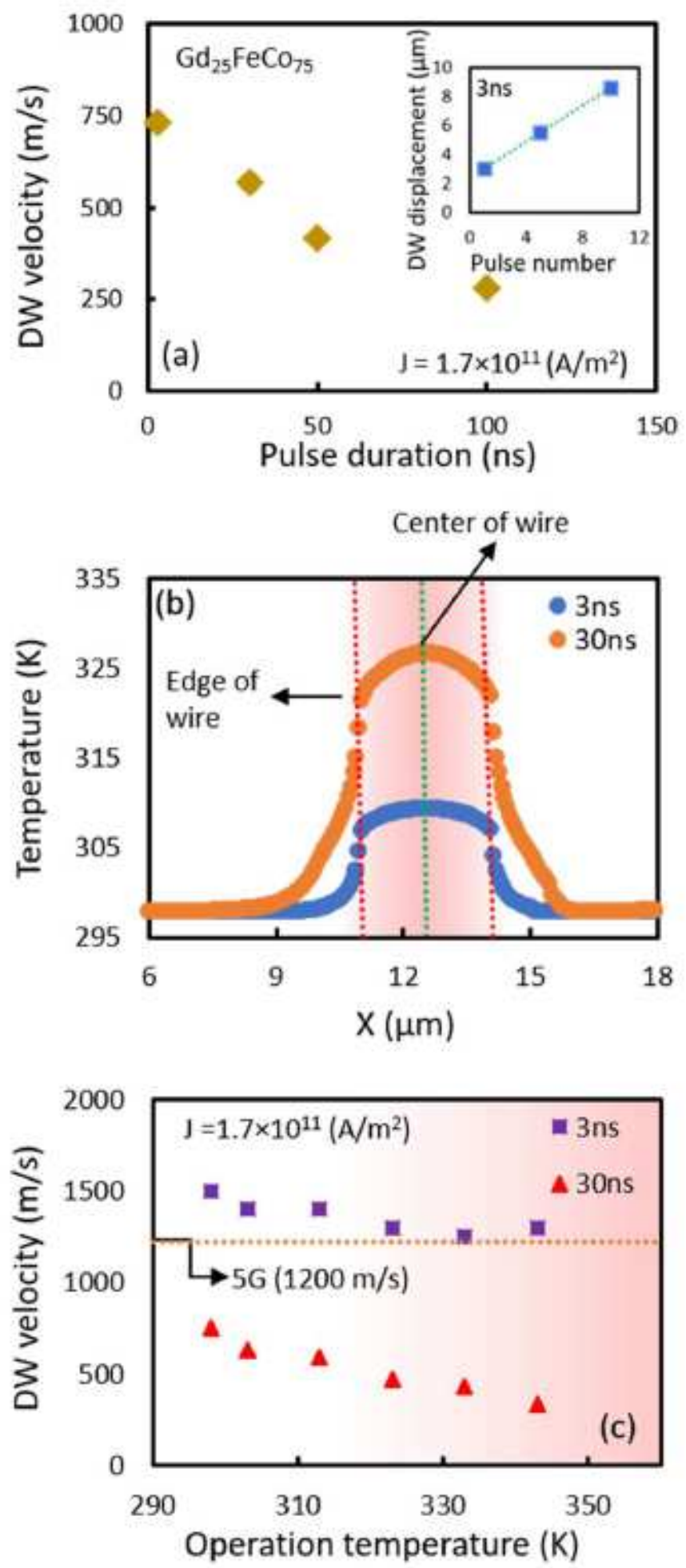

\section{Figure 4}

(a) the DW velocity as a function of pulse duration and the inset shows DW displacement as a function of pulse number (b) temperature profile after $t=30$ and $3 \mathrm{~ns}$ along the length of the wire on a silicon substrate (c) effect of operation temperature on DW speed for different pulse durations. 

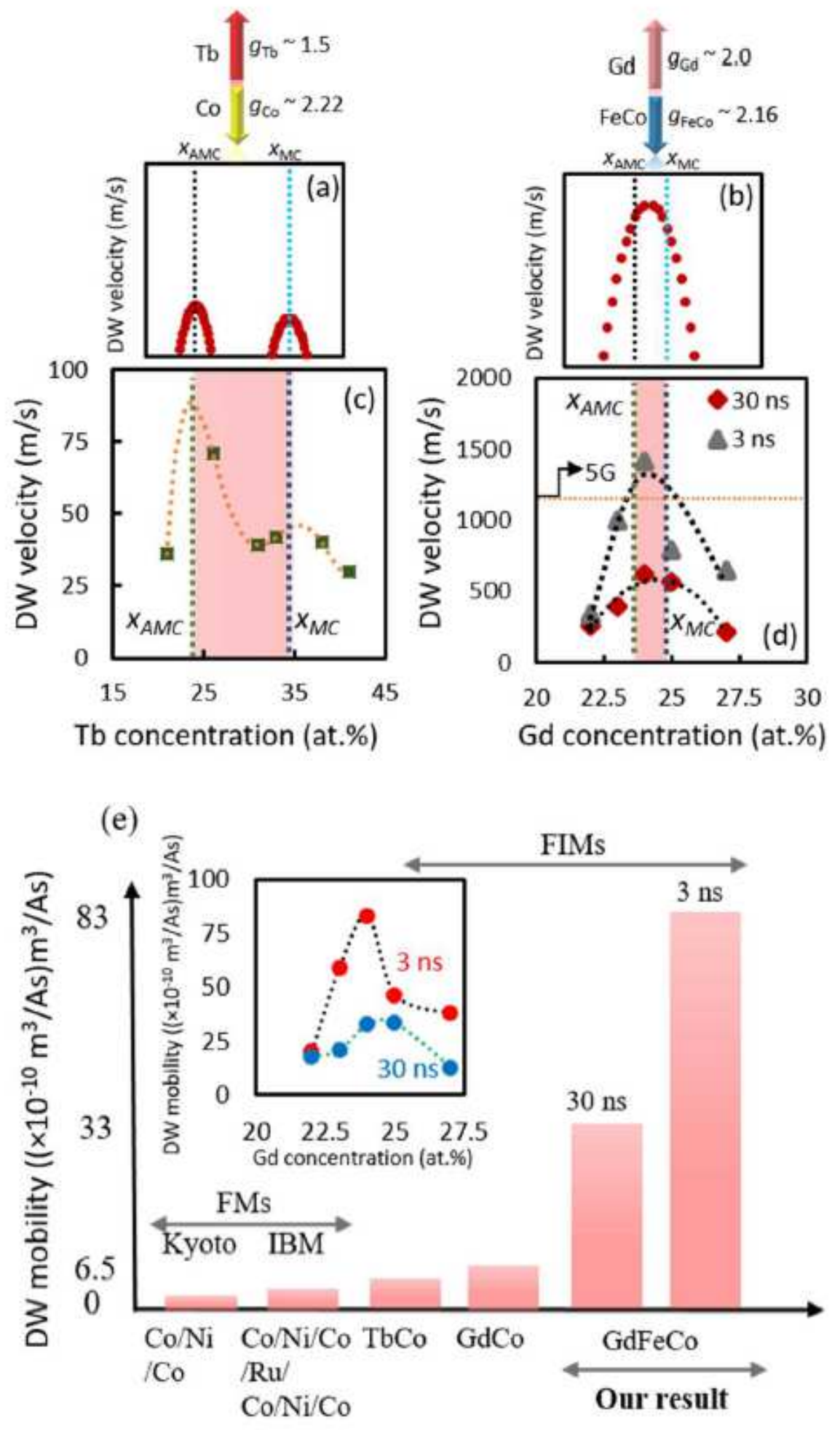

Figure 5

(a-b) schematic margin of TbCo and GdFeCo between angular momentum compensation composition and magnetic compensation composition (c-d) comparing TbCo 29 with our GdFeCo samples (e) comparison of DW mobility in our study with other reports.

\section{Supplementary Files}


This is a list of supplementary files associated with this preprint. Click to download.

- supl.pdf 